\title{
Correlative study of preoperative three-dimensional transvaginal ultrasound findings and ongoing pregnancy/live birth in patients with intrauterine adhesions following hysteroscopic adhesiolysis: a retrospective study
}

\author{
Xingping Zhao ${ }^{1}$, Yimin Yang ${ }^{1}$, Dan Liao $^{2}$, Absatou Traoré ${ }^{1}$, Sili $\mathrm{He}^{1}$, Dabao Xu ${ }^{1}$ \\ ${ }^{1}$ Department of Obstetrics and Gynecology, The Third Xiangya Hospital of Central South University, Changsha China; ${ }^{2}$ Department of Obstetrics \\ and Gynecology, The People's Hospital of Ningxiang, Ningxiang, China
}

Contributions: (I) Conception and design: D Xu, S He; (II) Administrative support: None; (III) Provision of study materials or patients: D Xu; (IV) Collection and assembly of data: X Zhao; (V) Data analysis and interpretation: X Zhao; (VI) Manuscript writing: All authors; (VII) Final approval of manuscript: All authors.

Correspondence to: Dabao Xu, PhD; Sili He, PhD. Department of Gynecology, Third Xiangya Hospital of Central South University, 138 Tongzipo Rd., Changsha 410013, China. Email: dabaoxu@yahoo.com; 986315943@qq.com.

Background: Three-dimensional transvaginal ultrasound (3D-TVUS) has recently been adopted in the gynecological sciences as it provides an accurate illustration of adhesions and the extent of cavity damage, and hence, can be used as an essential tool for the prognosis of intrauterine adhesions (IUA). This study aims to demonstrate whether preoperative 3D-TVUS features are relevant to ongoing pregnancy and live births in patients with IUA following hysteroscopic adhesiolysis (HA).

Methods: From February 22, 2018, to October 31, 2018, a total of 401 patients with moderate to severe IUA and underwent HA were retrospectively enrolled. Preoperative 3D-TVUS diagnosed data and the patients' basic information were collected, and patients were followed up over 2 years after HA for reproductive outcomes. The correlation between each imaging variable and ongoing pregnancy or live birth was analyzed by binary logistic regression.

Results: Among the 401 patients, 143 had live births, 41 patients had abortions, and 217 patients were infertile. Thick endometrium was found to be favorable for ongoing pregnancy (ongoing pregnancy group $=5.4 \pm 1.95 \mathrm{~mm}$, no-ongoing pregnancy group $=4.7 \pm 2.24 \mathrm{~mm}, \mathrm{P}=0.0095$ ) and live birth (live birth group $=5.6 \pm 1.92 \mathrm{~mm}$, no-live birth group $=4.7 \pm 2.20 \mathrm{~mm}, \mathrm{P}=0.0029)$. Scar contraction was not conducive for pregnancy, while the lower segmentation was not a risk factor for ongoing pregnancy $(\mathrm{P}=0.0003)$. It also was a risk factor for ongoing pregnancy $(\mathrm{P}<0.0001)$ and live birth $(\mathrm{P}<0.0001)$ when the segmentation of the endometrial absence was mainly in the upper and middle segments of the uterine cavity. The area under the curves (AUCs) of the prediction model for ongoing pregnancy and live birth were 0.9116 and 0.8751 , respectively, based on the meaningful variables above combined with other clinical characteristics.

Conclusions: Preoperative 3D-TVUS features have a close correlation with ongoing pregnancy and live births in patients with IUA following HA, and can be applied for predicting ongoing pregnancy and live births in IUA patients post-HA.

Keywords: Preoperative three-dimensional transvaginal ultrasound (preoperative 3D-TVUS); intrauterine adhesions (IUA); hysteroscopic adhesiolysis (HA)

Submitted Jul 14, 2021. Accepted for publication Dec 02, 2021; Published online: 24 Jan 2022.

doi: 10.21037/qims-21-727

View this article at: https://dx.doi.org/10.21037/qims-21-727 


\section{Introduction}

Intrauterine adhesions (IUAs) are scar/fibrous tissues that form between the inner walls of the uterus. Adhesions are believed to occur when contiguous traumatized uterine walls heal by abnormal fibrous secondary to trauma to a gravid, or non-gravid uterine cavity. It was inevitable that direct contact of these abraded areas where denudation or perforation of the myometrium would result in their coalescence by scarring. The main clinical presentations of IUA are menstrual abnormalities, secondary infertility/ subfertility, recurrent miscarriages (1), and pregnancy outcomes (2-5). Hysteroscopic adhesiolysis (HA) aims to restore the uterine cavity's volume and shape and enhance fecundity potential $(6,7)$. The characteristics of the endometrium are equally important for implantation. Various studies have reported that endometrial receptivity is an important component for successful blastocyst implantation. Endometrial receptivity is characterized as a momentary sole order of factors that make the endometrium receptive to the embryo's implantation; the time frame when the uterine milieu is favorable to blastocyst acquisition and the ensuing implantation. It has been reported that factors such as endometrial thickness, pattern and blood flow reflect endometrial receptivity $(6,8)$. The endometrium's other important features include the endometrial echo, peristalsis, volume, and endometrial-myometrial junctional zone (8). Early diagnosis and hysteroscopic treatment have undoubtedly been the key factors leading to improvements such as uterine mobility, visibility of the fallopian tube ostia, and normal uterine cavity size which are believed to influence pregnancy outcomes.

A variety of classifications of IUAs have been constructed in different countries to evaluate the severity of IUA, which are predominantly based on hysterosalpingography or hysteroscopic findings (9). Three-dimensional transvaginal ultrasound (3D-TVUS) has recently been adopted in the gynecological sciences as it enables multiplanar displays that help visualize the three orthogonal scan planes and obtain precise anatomical views of the uterine cavity. Compared with hysterosalpingography or hysteroscopic findings, $3 \mathrm{D}-\mathrm{TVUS}$ is a rapid and non-invasive technique that can be easily mastered without risk for patients. It provides a vivid $3 \mathrm{D}$ perspective of the endometrial cavity (10), and with the contribution of the coronal plane, a precise relationship between the cavity and the fundus can be established (11). 3D-TVUS provides an accurate illustration of adhesions and the extent of cavity damage, maybe, it can be used as an essential tool for IUA (6). 3D-TVUS can also be used to calculate the volume and study the vascularisation of the cavity, which can affect the prognosis of fertility.

This article aims to demonstrate whether preoperative 3D-TVUS features are relevant to pregnancy in patients with IUA following HA, especially to live birth, and to explore whether a prediction model can be established according to the 3D-TVUS features.

We present the following article in accordance with the STARD reporting checklist (available at https://qims. amegroups.com/article/view/10.21037/qims-21-727/rc).

\section{Methods}

\section{Patients}

This study collected IUA patients who had undergone preoperative $3 \mathrm{D}$-TVUS evaluation and HA between February 22, 2018 and October 31, 2018 to find out whether their pregnant outcomes were relevant to preoperative $3 \mathrm{D}-\mathrm{TVUS}$. The included patients had IUA (diagnosed by 3D-TVUS and hysteroscopy) and were excluded from having uterine anomalies and other reproductive system or systemic diseases. All patients had a strong desire to conceive. Preoperative 3D-TVUS data were retrospectively collected, and patients were followed up over 2 years after HA (for live birth, miscarriage, and infertility). Written informed consent for their medical records used for scientific research was signed voluntarily by each patient, and the ethics committee approved the study of the Third Xiangya Hospital of Central South University (IRB No. I-21046). The study was conducted in accordance with the Declaration of Helsinki (as revised in 2013)

The inclusion criteria were as follows: (I) patients with moderate to severe IUA (confirmed by hysteroscopy) according to the American Fertility Society (AFS) adhesion scoring system; (II) patients who had undergone 3D-TVUS evaluation before HA; (III) patients aged 18-45 years with a strong desire to conceive; and (IV) patients with normal hormone levels and ovulation in a natural and regular cycle. The exclusion criteria were as follows: (I) patients with cervical or endometrial malignant lesions; (II) patients with serious heart and liver conditions or renal insufficiency; (III) patients with serious nervous system diseases, who were unable to care for themselves in daily life or unable to undergo relevant treatment; (IV) patients with surgical intolerance or an inability to follow the doctor's advice to review or follow-up; (V) those with congenital malformation 

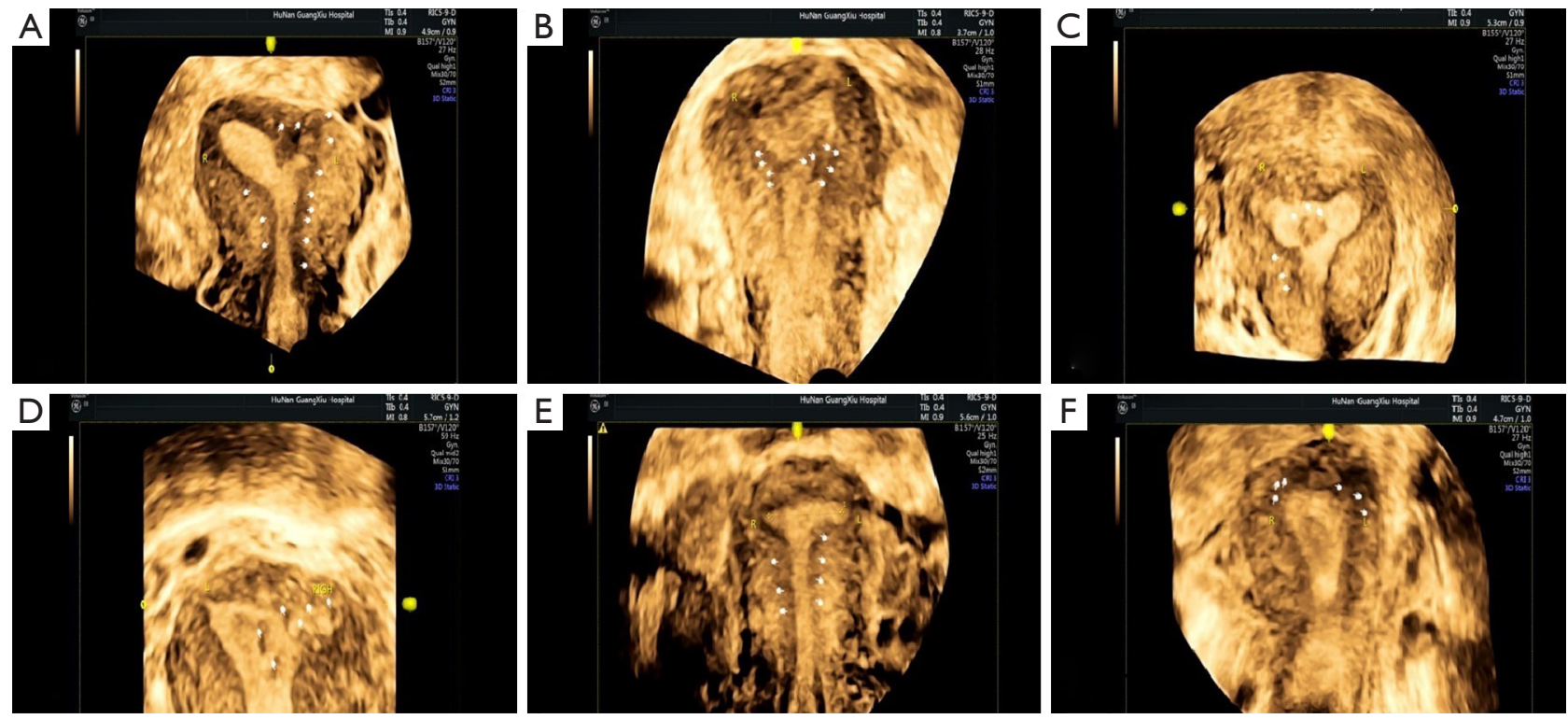

Figure 1 The morphological characteristics of intrauterine adhesions in three-dimensional transvaginal ultrasound imaging, little hands represent adhesion location. (A) Adhesions were located in the left uterine cavity and the lateral wall of the middle and lower segments. (B) Adhesions were located in the middle segment of uterine cavity. (C) Adhesions were located in the center of the uterine cavity and the right lateral wall. (D) Adhesions are located in the center of the uterine cavity and the left uterine cornua. (E) Adhesions were located in the middle and lower segments of uterine cavity. (F) Adhesions are located in bilateral uterine cornua.

of the uterus; and (VI) cases of male infertility.

A total of 413 patients met criteria and were included in the study. Patients were followed up over a period of 2 years after their hysteroscopy for live birth, miscarriage, and infertility (Ongoing pregnancy: yes means live birth and miscarriage, no means infertility; Live births: yes means live births, no means miscarriage, and infertility). Finally, 401 patients were followed up successfully by telephone.

\section{D-TVUS examination}

The GE VOLUSON E8 ultrasound instrument (USA) with a two-dimensional (2D) volume probe and realtime three-dimensional (3D) volume probe was used for preoperative 3D-TVUS examinations. Patients underwent preoperative 3D-TVUS during the secretory phase of the menstrual cycle, using a 7.5 MHz IC5-9D vaginal probe. During the examination, the patients emptied their bladder and were placed in the lithotomy position. Routine 2D ultrasonographic examinations were performed first. After rotating the real-time 3D volume probe, panoramic technology was used to obtain the overall image information and select the target area (Figure 1). Fixed probe in longitudinal section of the uterus, the best position to start the three-dimensional (3D) program, at the same time pay attention to adjusting the three-dimensional sampling volume. The scope includes the whole uterus and pay attention to make patients held her breath and press execute after $3 \mathrm{D}$ scanning measurement module of $3 \mathrm{D}$ reconstruction data. When complete image acquisition, the $3 \mathrm{D}$ reconstruction image can be got organized from the sagittal plane, cross sectional plane and coronary plane. With the uterine cavity as the center point, rotate the $\mathrm{X}$, $\mathrm{Y}$ and $\mathrm{Z}$ axes which are perpendicular to each other, and select the best observation angle, then the coronal plane 3D image of the uterus can be observed. On this image, we can observe whether the shape of uterine cavity is normal, defect, or the boundary between uterine cavity and muscle layer is clear, so as to judge whether there is IUA and the location of adhesion. Then the relevant uterine data were measured: the endometrial thickness, endometrial echo, endometrial blood flow, intercornual distance, visibility of the fallopian tubal ostia and so on. Endometrial blood flow can be judged by analyzing the hemodynamic parameters of uterine artery and spiral artery and the number and density of perfusion in the endometrial and subendometrial. There are four levels: level 0 (almost no blood flow signal in the endometrium), level 1 (blood flow only reaches 
the hyperechoic basal layer of the endometrium), level 2 (blood flow reaches the hypoechoic functional layer of the endometrium) and level 3 (blood flow reaches the central endometrium) When complete image acquisition, the 3D obtained and stored image can be got organized from the sagittal plane, cross sectional plane and coronary plane. OmniView, Render, MUltiplanar can be used as offline application for image adjustment and analysis. The 3D reconstruction image can be got organized from the sagittal plane, cross sectional plane and coronary plane. With the uterine cavity as the center point, rotate the $\mathrm{X}, \mathrm{Y}$ and $\mathrm{Z}$ axes which are perpendicular to each other, and select the best observation angle, then the coronal plane $3 \mathrm{D}$ image of the uterus can be observed. On this image, we can observe whether the shape of uterine cavity is normal, defect, or the boundary between uterine cavity and muscle layer is clear, so as to judge whether there is IUA and the location of adhesion. Then the relevant uterine data were measured: the endometrial thickness, endometrial echo, endometrial blood flow, intercornual distance, visibility of the fallopian tubal ostia, uterine artery [peak systolic flow rate/diastolic flow rate (S/D), resistant index (RI), and pulsatility index (PI)] and so on. Finally, the 3D-TVUS information was stored on a removable hard disk for further evaluation and calculation by two experienced senior sonographers.

\section{Statistical analysis}

SAS.9.4 statistical software (SAS, USA) was used to analyze the collected data. First, all of the variables were statistically described. Categorical measurements were described as counts and percentages, while continuous measurements were presented as means and ranges. Secondly, correlation analysis was conducted between the variables in the study and the ongoing pregnancy or live birth of IUA patients (Ongoing pregnancy: yes means live birth and miscarriage, no means infertility; Live births: yes means live births, no means miscarriage, and infertility). Spearman correlation was adopted for the continuous index, while the chisquare or Fisher's exact probability method was used for the discrete index. Logistic regression was used to explore the correlation between ongoing pregnancy (or live birth) and Preoperative 3D-TVUS features. To establish a model for predicting ongoing pregnancy and live birth in IUA patients post-HA, multivariate logistical regression analysis was carried out based on the meaningful variables $(\mathrm{P}<0.05)$ identified by univariate logistical regression analysis. A P value $<0.05$ was considered statistically significant.

\section{Results}

\section{Related factors affecting the ongoing pregnancy or live birth of IUA patients post-HA}

So far, the success rate of $3 \mathrm{D}$ TVUS is 100 percent. Correlation analysis was conducted between the variables and the ongoing pregnancy or live birth of IUA patients (shown in Table 1). The endometrial thickness ( $\mathrm{mm}$ ), intercornual distance ( $\mathrm{mm}$ ), endometrial echo, segmentation of scar contraction, segmentation of the endometrial loss, visibility of fallopian tube ostia, and endometrial blood flow were closely related to ongoing pregnancy and live birth $(\mathrm{P}<0.05)$. Other variables, including pregnancy history and uterine artery (S/D, RI, and PI), did not exhibit any statistical significance related to ongoing pregnancy and live birth post-HA $(\mathrm{P}>0.05)$.

\section{Univariate logistic regression analysis of the related factors}

Logistic regression was used to explore the influencing factors of ongoing pregnancy or live birth of IUA patients (Table 2). The intercornual distance was wider in the ongoing pregnancy and live birth groups $[\mathrm{P}=0.0031$, odds ratio $(\mathrm{OR})=1.065,95 \% \mathrm{CI}: 1.021-1.11$ and $\mathrm{P}=0.0105$, OR $=1.055,95 \%$ CI: 1.013-1.099, respectively]. Endometrial thickness in the ongoing pregnancy and live birth groups was greater than in the non-ongoing pregnancy and non-live birth groups $(\mathrm{P}=0.0007, \mathrm{OR}=1.192,95 \% \mathrm{CI}$ : $1.076-1.32$ and $\mathrm{P}=0.0004$, $\mathrm{OR}=1.204$, $95 \% \mathrm{CI}: 1.087-$ 1.334 , respectively). The endometrial echo was more homogeneous in the ongoing pregnancy and live birth groups $(\mathrm{P}=0.0002$, OR $=0.456,95 \% \mathrm{CI}: 0.304-0.685$ and $\mathrm{P}<0.0001, \mathrm{OR}=0.421,95 \% \mathrm{CI}: 0.277-0.64$, respectively). The segmentation of scar contraction in the ongoing pregnancy group was mainly in the middle and lower segments of the uterine cavity $(\mathrm{P}=0.0061, \mathrm{OR}=0.359$, 95\% CI: $0.173-0.747$ and $\mathrm{P}=0.0008, \mathrm{OR}=2.121,95 \%$ CI: 1.366-3.294, respectively). The segmentation of the endometrial absence in the ongoing pregnancy group was mainly in middle and lower segments of the uterine cavity $(\mathrm{P}=0.0001, \mathrm{OR}=2.647,95 \% \mathrm{CI}: 1.617-4.335$ and $\mathrm{P}=0.0034$, OR $=6.667,95 \%$ CI: $1.874-23.721$, respectively), while that of the non-ongoing pregnancy group was primarily in the upper segment $(\mathrm{P}<0.0001, \mathrm{OR}=0.113,95 \%$ CI: 0.053-0.24). The segmentation of scar contraction and segmentation of the endometrial absence in the live birth group exhibited the same phenomenon. The number of visible fallopian tube ostia was greater in the ongoing pregnancy and live birth 
Table 1 Related factors affecting pregnancy or live birth in IUA patients post-HA

\begin{tabular}{|c|c|c|c|c|c|c|c|c|c|}
\hline $\begin{array}{l}\text { Clinical } \\
\text { characteristics }\end{array}$ & Category & \multicolumn{4}{|c|}{ Ongoing pregnancy } & \multicolumn{4}{|c|}{ Live birth } \\
\hline \multirow[t]{3}{*}{ Age } & $\mathrm{N}$ [Nmiss] & $184[0]$ & $217[0]$ & 0.053 & 0.2901 & $143[0]$ & $258[0]$ & 0.064 & 0.2008 \\
\hline & Mean (SD) & $31.4(5.65)$ & $31.9(5.03)$ & & & $31.2(5.59)$ & $31.9(5.16)$ & & \\
\hline & Median & 30 & 31 & & & 30 & 31 & & \\
\hline \multirow{4}{*}{ Gravidity } & 1 & $41(22.3 \%)$ & $51(23.5 \%)$ & & & $32(22.4 \%)$ & $60(23.3 \%)$ & & \\
\hline & 2 & $66(35.9 \%)$ & $59(27.2 \%)$ & & & $52(36.4 \%)$ & $73(28.3 \%)$ & & \\
\hline & 3 & $23(12.5 \%)$ & $41(18.9 \%)$ & & & $21(14.7 \%)$ & $43(16.7 \%)$ & & \\
\hline & $\geq 4$ & $49(26.6 \%)$ & $61(28.1 \%)$ & & & $38(26.5 \%)$ & $72(27.8 \%)$ & & \\
\hline \multirow{6}{*}{ Parity } & 0 & $113(61.4 \%)$ & $122(56.2 \%)$ & & & $93(65.0 \%)$ & $142(55.0 \%)$ & & \\
\hline & 1 & $58(31.5 \%)$ & 78 (35.9\%) & & & 45 (31.5\%) & $91(35.3 \%)$ & & \\
\hline & 2 & $6(3.3 \%)$ & $11(5.1 \%)$ & & & $4(2.8 \%)$ & $13(5.0 \%)$ & & \\
\hline & 3 & $1(0.5 \%)$ & $1(0.5 \%)$ & & & $0(0.0 \%)$ & $2(0.8 \%)$ & & \\
\hline & 4 & $1(0.5 \%)$ & $0(0.0 \%)$ & & & $1(0.7 \%)$ & $0(0.0 \%)$ & & \\
\hline & Total & $184(100.0 \%)$ & 217 (100.0\%) & & & 143 (100.0\%) & $258(100.0 \%)$ & & \\
\hline \multirow[t]{2}{*}{ Abortion } & Nmiss & $5(2.7 \%)$ & $5(2.3 \%)$ & 0.0236 & 0.6419 & $0(0.0 \%)$ & $10(3.9 \%)$ & 0.0108 & 0.8315 \\
\hline & 0 & $6(3.3 \%)$ & $5(2.3 \%)$ & & & $5(3.5 \%)$ & $6(2.3 \%)$ & & \\
\hline \multirow{6}{*}{$\begin{array}{l}\text { Number of } \\
\text { curettage in } \\
\text { artificial abortion }\end{array}$} & Nmiss & $5(2.7 \%)$ & $5(2.3 \%)$ & -0.0155 & 0.7594 & $0(0.0 \%)$ & $10(3.9 \%)$ & 0.0137 & 0.7876 \\
\hline & 1 & $114(62.0 \%)$ & $142(65.4 \%)$ & & & $93(65.0 \%)$ & $163(63.2 \%)$ & & \\
\hline & 2 & $43(23.4 \%)$ & 36 (16.6\%) & & & $34(23.8 \%)$ & 45 (17.4\%) & & \\
\hline & 3 & $10(5.4 \%)$ & $13(6.0 \%)$ & & & $9(6.3 \%)$ & $14(5.4 \%)$ & & \\
\hline & $\geq 4$ & 12 (6.5\%) & $21(9.7 \%)$ & & & 7 (4.9\%) & $26(10.1 \%)$ & & \\
\hline & Total & 184 (100.0\%) & 217 (100.0\%) & & & $143(100.0 \%)$ & 258 (100.0\%) & & \\
\hline \multirow{3}{*}{$\begin{array}{l}\text { Endometrial } \\
\text { thickness (mm) }\end{array}$} & $\mathrm{N}$ [Nmiss] & $181[3]$ & 202 [15] & -0.2062 & 0.0000 & $141[2]$ & $242[16]$ & -0.2237 & 0.0000 \\
\hline & Mean (SD) & $5.4(1.95)$ & $4.7(2.24)$ & & & $5.6(1.92)$ & $4.7(2.20)$ & & \\
\hline & Median & 5.2 & 4.4 & & & 5.3 & 4.4 & & \\
\hline
\end{tabular}

Table 1 (continued) 
Table 1 (continued)

\begin{tabular}{|c|c|c|c|c|c|c|c|c|c|}
\hline $\begin{array}{l}\text { Clinical } \\
\text { characteristics }\end{array}$ & Category & \multicolumn{4}{|c|}{ Ongoing pregnancy } & \multicolumn{4}{|c|}{ Live birth } \\
\hline \multirow{3}{*}{$\begin{array}{l}\text { Intercornual } \\
\text { distance (mm) }\end{array}$} & $\mathrm{N}$ [Nmiss] & $167[17]$ & 189 [28] & -0.1731 & 0.001 & $131[12]$ & 225 [33] & -0.1532 & 0.0038 \\
\hline & Mean (SD) & $25.42(5.738)$ & 23.68 (4.910) & & & $25.47(5.802)$ & $23.93(5.040)$ & & \\
\hline & Median & 25 & 23 & & & 25 & 23 & & \\
\hline \multirow{2}{*}{$\begin{array}{l}\text { Endometrial } \\
\text { echo }\end{array}$} & Uneven & $91(49.5 \%)$ & $148(68.2 \%)$ & & & $66(46.2 \%)$ & 173 (67.1\%) & & \\
\hline & Total & $184(100.0 \%)$ & 217 (100.0\%) & & & 143 (100.0\%) & 258 (100.0\%) & & \\
\hline \multirow{3}{*}{$\begin{array}{l}\text { Segmentation of } \\
\text { scar contraction }\end{array}$} & No & $70(38.0 \%)$ & $96(44.2 \%)$ & -0.1668 & 0.0008 & $53(37.1 \%)$ & $113(43.8 \%)$ & -0.1371 & 0.006 \\
\hline & Upper & $4(2.2 \%)$ & $15(6.9 \%)$ & & & $4(2.8 \%)$ & $15(5.8 \%)$ & & \\
\hline & Total & $184(100.0 \%)$ & 217 (100.0\%) & & & 143 (100.0\%) & 258 (100.0\%) & & \\
\hline \multirow{5}{*}{$\begin{array}{l}\text { Segmentation of } \\
\text { the endometrial } \\
\text { absence }\end{array}$} & No & $68(37.0 \%)$ & $80(36.9 \%)$ & -0.2291 & 0.0000 & $54(37.8 \%)$ & 94 (36.4\%) & -0.1728 & 0.0005 \\
\hline & Upper & $9(4.9 \%)$ & $94(43.3 \%)$ & & & $8(5.6 \%)$ & $95(36.8 \%)$ & & \\
\hline & Middle & $90(48.9 \%)$ & 40 (18.4\%) & & & $66(46.2 \%)$ & $64(24.8 \%)$ & & \\
\hline & Lower & $17(9.2 \%)$ & $3(1.4 \%)$ & & & $15(10.5 \%)$ & $5(1.9 \%)$ & & \\
\hline & Total & $184(100.0 \%)$ & 217 (100.0\%) & & & 143 (100.0\%) & 258 (100.0\%) & & \\
\hline \multirow{2}{*}{$\begin{array}{l}\text { Visibility of } \\
\text { fallopian tube } \\
\text { ostia }\end{array}$} & Nmiss & $90(48.9 \%)$ & $93(42.9 \%)$ & 0.2554 & 0.0001 & $66(46.2 \%)$ & 117 (45.3\%) & 0.2411 & 0.0003 \\
\hline & Bilateral visible & 55 (29.9\%) & $46(21.2 \%)$ & & & $46(32.2 \%)$ & 55 (21.3\%) & & \\
\hline \multirow{5}{*}{$\begin{array}{l}\text { Endometrial } \\
\text { blood flow }\end{array}$} & Nmiss & $4(2.2 \%)$ & $10(4.6 \%)$ & -0.1355 & 0.0076 & $4(2.8 \%)$ & $10(3.9 \%)$ & -0.1517 & 0.0028 \\
\hline & 0 degree & $42(22.8 \%)$ & 71 (32.7\%) & & & 30 (21.0\%) & $83(32.2 \%)$ & & \\
\hline & 1 degree & $124(67.4 \%)$ & 128 (59.0\%) & & & $96(67.1 \%)$ & 156 (60.5\%) & & \\
\hline & 2 degree & $14(7.6 \%)$ & $8(3.7 \%)$ & & & $13(9.1 \%)$ & 9 (3.5\%) & & \\
\hline & Total & 184 (100.0\%) & 217 (100.0\%) & & & 143 (100.0\%) & 258 (100.0\%) & & \\
\hline \multirow{3}{*}{$\begin{array}{l}\text { Left uterine } \\
\text { artery S/D }\end{array}$} & $\mathrm{N}$ [Nmiss] & $133[51]$ & $166[51]$ & 0.0585 & 0.3135 & $102[41]$ & 197 [61] & -0.0076 & 0.8964 \\
\hline & Mean (SD) & 4.943 (1.9322) & $5.210(2.1674)$ & & & $5.092(2.0343)$ & $5.091(2.0888)$ & & \\
\hline & Median & 4.72 & 4.84 & & & 4.845 & 4.81 & & \\
\hline
\end{tabular}

Table 1 (continued) 
Table 1 (continued)

\begin{tabular}{|c|c|c|c|c|c|c|c|c|c|}
\hline $\begin{array}{l}\text { Clinical } \\
\text { characteristics }\end{array}$ & Category & \multicolumn{4}{|c|}{ Ongoing pregnancy } & \multicolumn{4}{|c|}{ Live birth } \\
\hline \multirow{3}{*}{$\begin{array}{l}\text { Left uterine } \\
\text { artery RI }\end{array}$} & $\mathrm{N}$ [Nmiss] & $133[51]$ & $166[51]$ & 0.0597 & 0.3032 & $102[41]$ & 197 [61] & -0.0125 & 0.8299 \\
\hline & Mean (SD) & $0.797(0.2752)$ & $0.776(0.1588)$ & & & $0.811(0.3097)$ & $0.772(0.1498)$ & & \\
\hline & Median & 0.79 & 0.8 & & & 0.79 & 0.79 & & \\
\hline \multirow{2}{*}{$\begin{array}{l}\text { Left uterine } \\
\text { artery PI }\end{array}$} & Mean (SD) & $1.755(0.5445)$ & $1.868(0.5813)$ & & & $1.761(0.5659)$ & $1.847(0.5669)$ & & \\
\hline & Median & 1.725 & 1.89 & & & 1.72 & 1.865 & & \\
\hline \multirow{2}{*}{$\begin{array}{l}\text { Right uterine } \\
\text { artery S/D }\end{array}$} & $\mathrm{N}$ [Nmiss] & $133[51]$ & $166[51]$ & -0.0049 & 0.9326 & $102[41]$ & 197 [61] & -0.0595 & 0.3048 \\
\hline & Mean (SD) & $5.010(1.9140)$ & $5.072(2.2190)$ & & & $5.156(2.0251)$ & $4.986(2.1191)$ & & \\
\hline \multirow{2}{*}{$\begin{array}{l}\text { Right uterine } \\
\text { artery RI }\end{array}$} & Mean (SD) & $0.814(0.2730)$ & $0.765(0.1563)$ & & & $0.830(0.3079)$ & $0.765(0.1462)$ & & \\
\hline & Median & 0.78 & 0.79 & & & 0.8 & 0.79 & & \\
\hline \multirow{3}{*}{$\begin{array}{l}\text { Right uterine } \\
\text { artery PI }\end{array}$} & $\mathrm{N}$ [Nmiss] & $130[54]$ & $162[55]$ & 0.0366 & 0.5335 & $99[44]$ & $193[65]$ & -0.0055 & 0.9255 \\
\hline & Mean (SD) & $1.779(0.5421)$ & $1.812(0.5988)$ & & & $1.800(0.5779)$ & $1.796(0.5728)$ & & \\
\hline & Median & 1.685 & 1.85 & & & 1.71 & 1.82 & & \\
\hline
\end{tabular}

IUA, intrauterine adhesions; HA, hysteroscopic adhesiolysis; SD, standard deviation; P1: P value for pregnancy (Yes vs. No); P2: P value for live birth (Yes vs. No); RI, resistance index; PI, pulsatility index; S/D, peak systolic flow rate/diastolic flow rate; r, correlation coefficients.

Table 2 Univariate logistic regression analysis of related factors

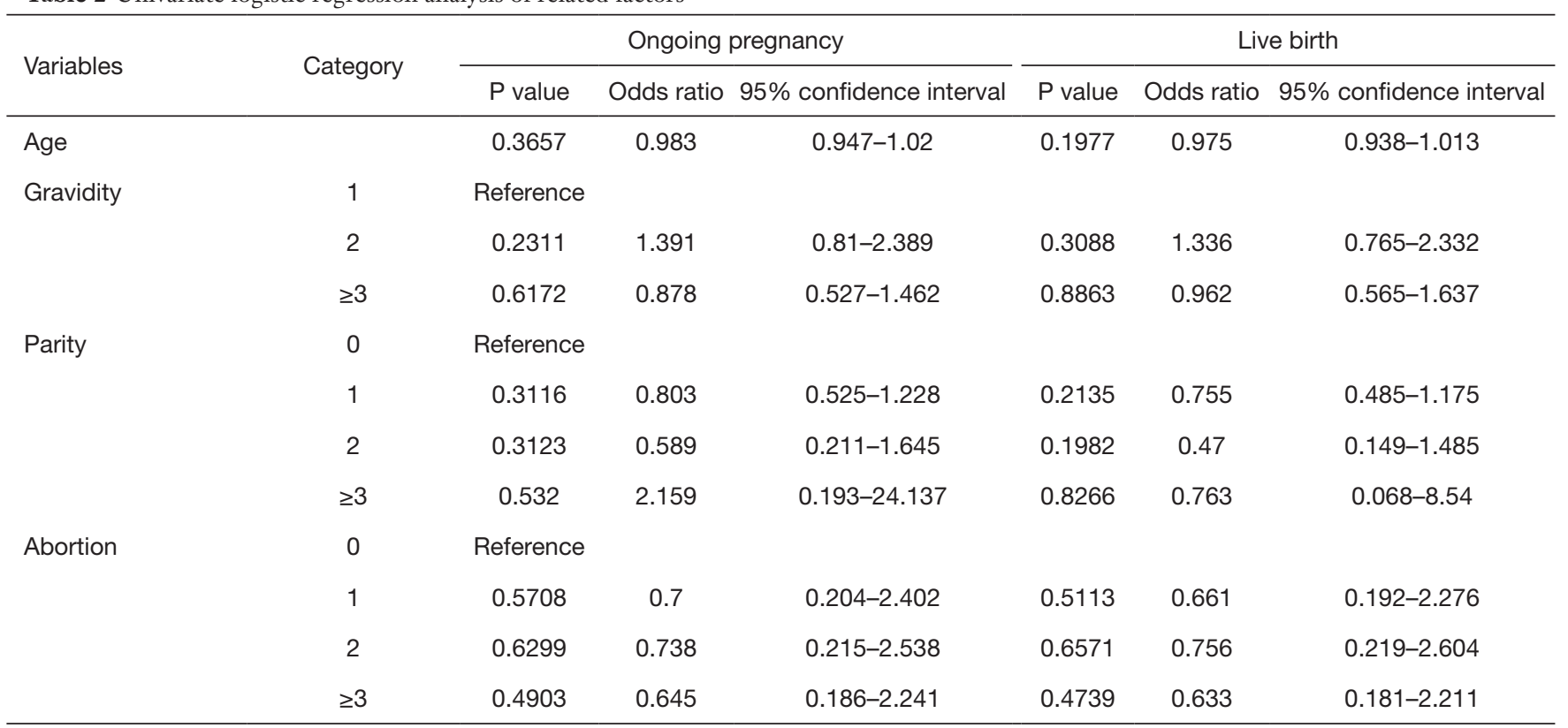

Table 2 (continued) 
Table 2 (continued)

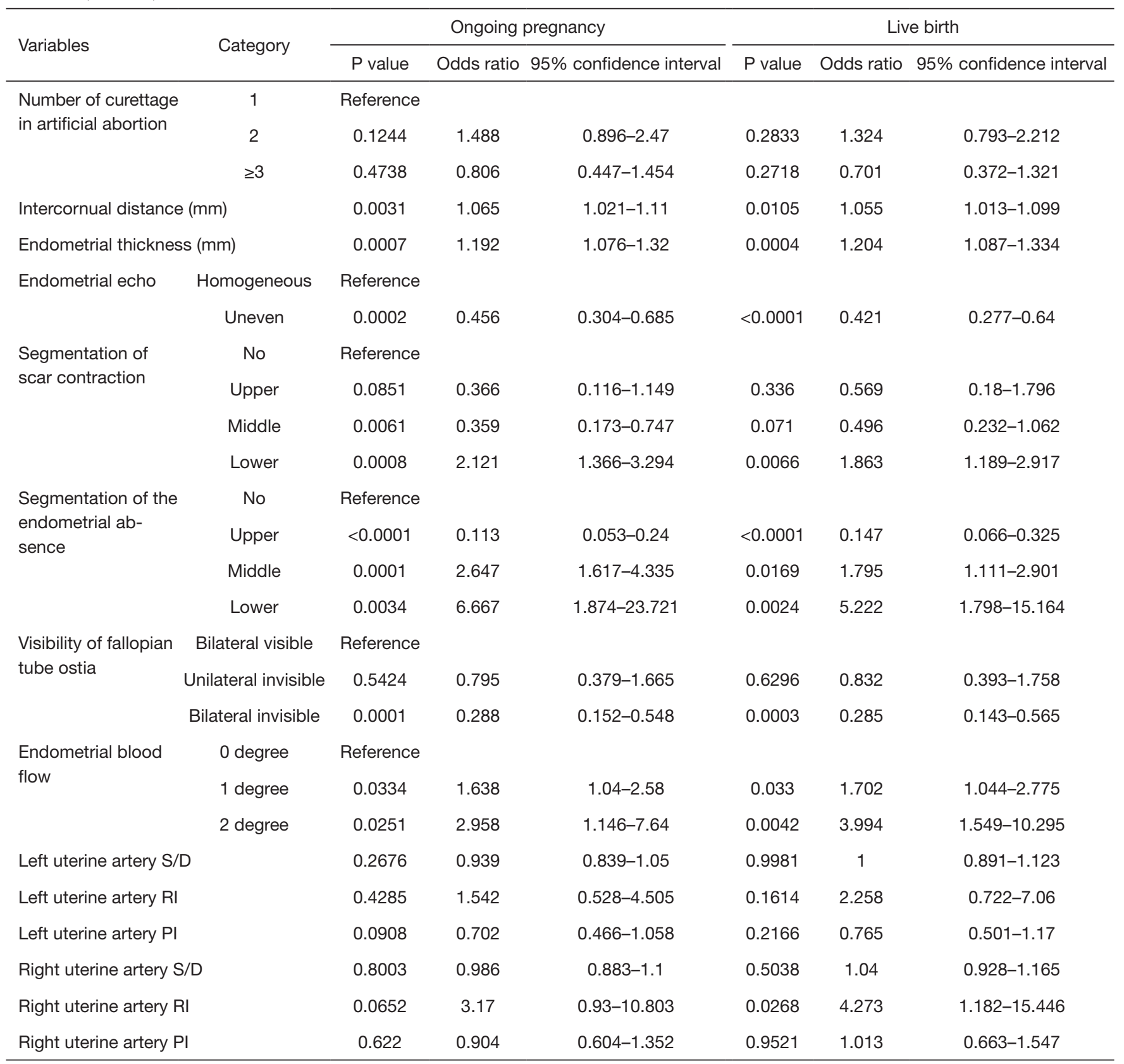

$\mathrm{RI}$, resistance index; PI, pulsatility index; S/D, peak systolic flow rate/diastolic flow rate.

groups $(\mathrm{P}=0.0001, \mathrm{OR}=0.288,95 \% \mathrm{CI}: 0.152-0.548$ and $\mathrm{P}=0.0003, \mathrm{OR}=0.285,95 \%$ CI: $0.143-0.565$, respectively). Endometrial blood flow was also better in the ongoing pregnancy and live birth groups ( 2 degree: $\mathrm{P}=0.0251$, OR $=2.958,95 \%$ CI: $1.146-7.64$ and $\mathrm{P}=0.0042, \mathrm{OR}=3.994$, 95\% CI: $1.549-10.295)$.

\section{Prediction model of ongoing pregnancy and live birth in IUA patients post-HA}

Thick endometrium was found to be favorable for ongoing pregnancy $(\mathrm{P}=0.0095, \mathrm{OR}=1.166,95 \% \mathrm{CI}$ : $1.038-1.309)$. Scar contraction of the upper segmentation 
Table 3 Prediction model of ongoing pregnancy and live birth in IUA patients post-HA

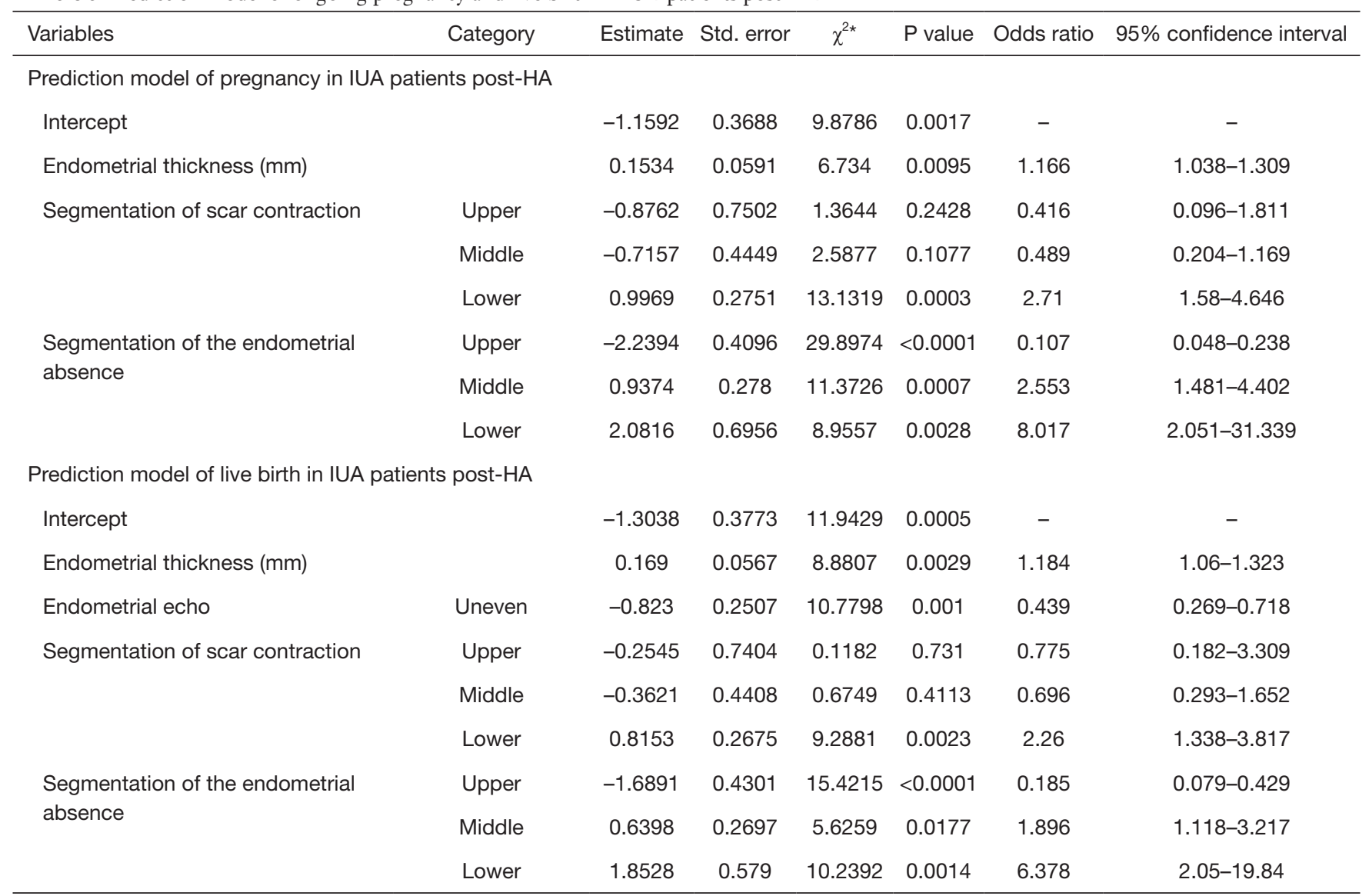

*, chi-square test for entire group. IUA, intrauterine adhesions; HA, hysteroscopic adhesiolysis.

was not conducive to ongoing pregnancy, while the lower segmentation was not a risk factor for ongoing pregnancy $(\mathrm{P}=0.0003, \mathrm{OR}=2.71,95 \%$ CI: 1.58-4.646). Similarly, we found that it was a risk factor for ongoing pregnancy when the segmentation of the endometrial absence was mainly in upper and middle segments of the uterine cavity $(\mathrm{P}<0.0001$, $\mathrm{OR}=0.107,95 \%$ CI: $0.048-0.238$ and $\mathrm{P}=0.0007$, OR $=2.553,95 \%$ CI: $1.481-4.402$, respectively) (Table 3). The area under the curve (AUC) of the prediction model for ongoing pregnancy was 0.9116 , based on the meaningful variables above combined with other clinical characteristics (Figure 2). The risk factors for live birth were same as those for ongoing pregnancy, including thinner endometrium $(\mathrm{P}=0.0029, \mathrm{OR}=1.184,95 \% \mathrm{CI}: 1.06-1.323)$, upper and middle segmentation of the endometrial absence $(\mathrm{P}<0.0001$, $\mathrm{OR}=0.185,95 \%$ CI: $0.079-0.429$ and $\mathrm{P}=0.0177$, OR $=1.896,95 \%$ CI: $1.118-3.217$, respectively), and uneven endometrial echo $(\mathrm{P}=0.001$, OR $=0.439,95 \%$ CI: 0.269 -
0.718). The AUC of the prediction model for live birth was 0.8751 , based on the meaningful variables above combined with other clinical characteristics (Figure 3).

\section{Discussion}

There are seven reported classification systems to categorize IUA (12-17). Traditionally, this classification was based on hysterosalpingography findings (18), but currently, numerous classifications have been proposed based on hysteroscopic findings $(19,20)$. Kim et al. first proposed a classification for IUA based on 3D-TVUS findings (10). But there was no literature so far on correlations between preoperative 3D TVUS and hysteroscopic evaluation. In this study, we found that Preoperative 3D-TVUS features are strongly correlated with ongoing pregnancy and live birth in IUA patients after HA.

With the development of ultrasonography, 3D-TVUS 


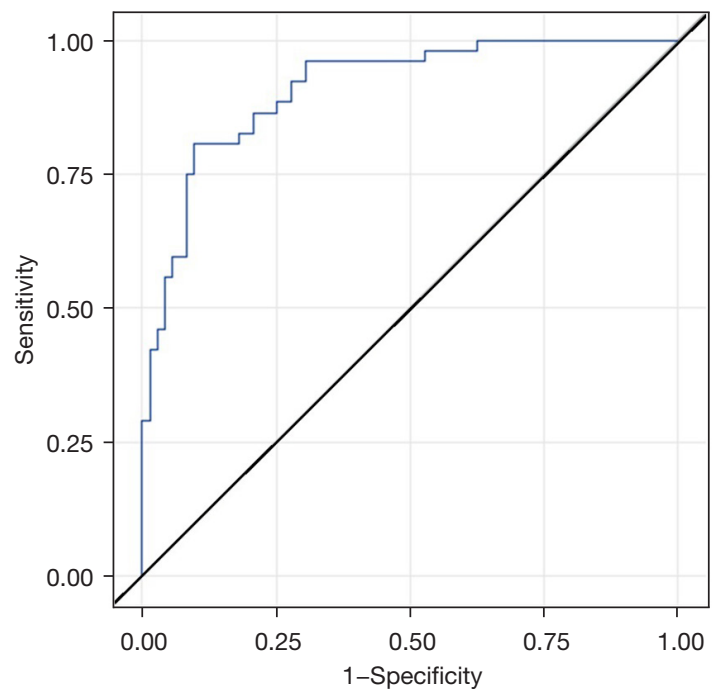

Figure 2 Receiver operating characteristic (ROC) curve of the prediction model for pregnancy. The area under the curve (AUC) of the prediction model based on the meaningful variables of three-dimensional transvaginal ultrasound imaging features for pregnancy was 0.9116 .

has been more frequently used in the field of obstetrics and gynecology (21). 3D reconstruction of the uterine anatomy provides panoramic views of the uterine cavity in the coronal plane. The narrowing or "bands" adherent across the cavity is usually well delineated on the coronal plane on $3 \mathrm{D}$ imaging. By acquiring a set volume that is stored, volumetric acquisitions allow for the offline review, manipulation, and analysis of saved images to obtain maximum information from a study. Knopman et al. stated that $3 \mathrm{D}$ ultrasound provides more accurate visualization of the adhesions and extent of cavity destruction than 2D ultrasound, with a sensitivity of $100 \%$ (22). Yan et al. concluded that the diagnostic accuracy $(87.5 \%)$, sensitivity (97\%), and specificity $(66.7 \%)$ of $3 \mathrm{D}$ ultrasonography were good in comparison with those of hysteroscopy (2). Another advantage of 3D-TVUS over hysteroscopy is that the latter cannot diagnose IUA in cases of intrauterine fibrosis without visible adhesion or obliteration of the cavity, whereas the former can (23). 3D-TVUS may be useful when hysteroscopy fails. Sometimes, the hysteroscope cannot simply access the cervical canal or uterine cavity due to dense adhesions. From the data obtained via 3D-TVUS, the surgeon could anticipate what to see and where to expect adhesions. The absence of an expected finding in a specific area or segment might indicate that the hysteroscope is not

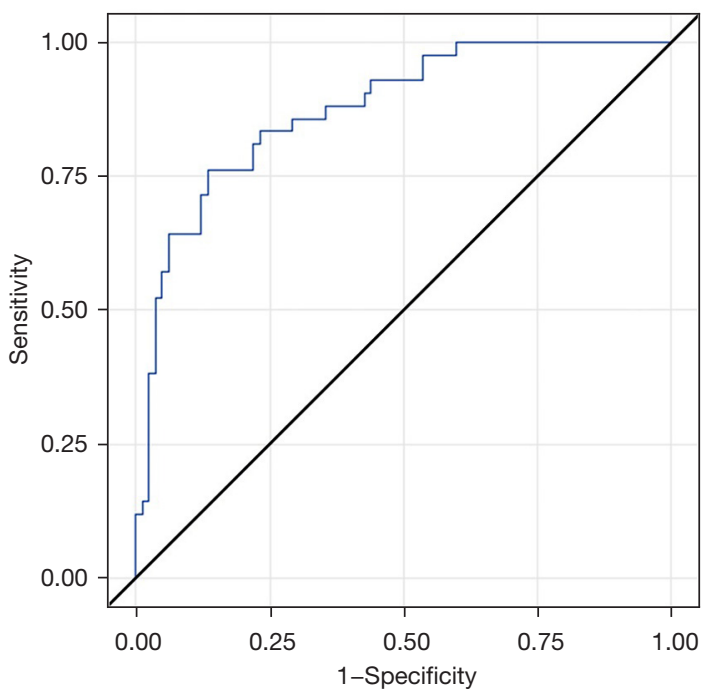

Figure 3 Receiver operating characteristic (ROC) curve of the prediction model for live birth. The area under the curve (AUC) of the prediction model based on the meaningful variables of threedimensional transvaginal ultrasound imaging features for live birth was 0.8751 .

in the proper layer or location.

In addition to factors described by Kim et al., who proposed a classification using $3 \mathrm{D}$-TVUS findings that differentiated six categories of IUA based on morphological characteristics of the endometrium (24), we conducted a review of all factors that could influence the prognosis of IUA and elaborated on the possible role of 3D-TVUS in predicting ongoing pregnancy outcomes of IUA. The extent of IUA has long been regarded as an important factor determining reproductive outcomes and has already been included in most of the classification systems (25). The smaller the proportion of the affected area, the better the prognosis of IUA. The location of IUA is associated with postoperative adhesion reformation potential. There is an increased possibility of IUA recurrence when adhesions are located at the uterine cornua (and specifically at the tubal ostium) (9), the cervico-isthmic region, and when it involves a large portion of the uterine cavity (26). Therefore, clear cornual angles and visibility of the fallopian tube ostia are considered important prognostic factors for successful HA.

Since IUA treatment is homogeneous, endometrial damage in IUA patients is mostly irreversible, we believe that preoperative $3 \mathrm{D}$-TVUS can also reflect the ongoing pregnancy outcome to a certain extent. Preoperative 3D-TVUS has the ability and accuracy in measuring and 
assessing the endometrial thickness, echo, and blood flow indices and the intercornual distance (suggestive of the volume of the endometrium), visibility of cornual angles, and fallopian tube ostia. Some scholars have reported the value of endometrial thickness, pattern, and blood flow in terms of pregnancy outcomes for in vitro fertilization (23). We studied these ultrasonographic indices to identify a relationship between the features of preoperative 3D-TVUS and pregnancy outcomes after HA. We found that the intercornual distance was wider, the endometrial thickness was greater and the endometrial echo was more homogeneous in the ongoing pregnancy and live birth groups. Of course, the shortage of the method used in this study was that endometrial volume can't be obtained and stored for offline evaluation.

A previous report found depositing embryos in the uterine mid-fundal area to be valuable in improving ongoing pregnancy rates (24). In our study, the segmentation of the endometrial absent and scar contraction in upper and middle segmentation of uterine cavity were high-risk factors for ongoing pregnancy and live birth. A possible reason is that the fundal endometrium is suitable for implantation as there is a tendency to a lower endometrial wavelike activity and higher endometrial tissue blood flow in the fundal endometrium (27). IUA in the upper uterine cavity segment, particularly in the fundal area destroyed the most suitable implanting site which might have resulted in a decrease of ongoing pregnancy rates.

Women prone to IUA must be encouraged to undergo this examination at an early stage of the disease. 3D-TVUS can describe the location, extent, and severity of the adhesions and confirm the presence and extent of a healthy endometrium so that a diagnostic hysteroscopy would less likely be needed to evaluate the prognosis of IUA (28-30). The limited success of any treatment might be related to the underlying disease, such as endometrial fibrosis. Hence, restoration of a normal uterine cavity and regular menstruation is not inevitably followed by normal fertility $(6,31)$. The limitation of this study is that there is no postoperative 3D-TVUS. As 3D-TVUS post-operatively is important to evaluate the operation result, postoperative 3D-TVUS should be recommended to IUA patients and the association between postoperative 3D-TVUS and pregnancy outcomes should be investigated in the future. 3D-TVUS can also be performed postoperatively to assess the intrauterine cavity, thereby avoiding unnecessary early second-look hysteroscopy. To the best of our knowledge, there is no documented literature on the use of preoperative
$3 \mathrm{D}$-TVUS in the prognosis of IUA and subsequent pregnancy outcomes. It showed an excellent performance in predicting ongoing pregnancy ( $\mathrm{AUC}=0.9116$ ) and live birth (AUC $=0.8751$ ) that the 3D-TVUS features combined with other clinical characteristics.

\section{Conclusions}

Preoperative 3D-TVUS features are strongly correlated with ongoing pregnancy and live birth in IUA patients after $\mathrm{HA}$, and more prospective investigations should be done to show that it can predict ongoing pregnancy and live birth.

\section{Acknowledgments}

Funding: This study was supported by the Natural Science Foundation of Hunan Provincial (No. 2021JJ40956 and No. 2020JJ5851), the Hunan Science and Technology Department (No. 2020 SK4017), National Key Research and Development Program of China (No. 2018YFC1004800) and the Hunan Provincial Clinical Medical Technology Innovation Guiding Project (No. 2020SK53605).

\section{Footnote}

Reporting Checklist: The authors have completed the STARD reporting checklist. Available at https://qims.amegroups. com/article/view/10.21037/qims-21-727/rc

Conflicts of Interest: All authors have completed the ICMJE uniform disclosure form (available at https://qims. amegroups.com/article/view/10.21037/qims-21-727/coif). All authors report that this study was supported by the Natural Science Foundation of Hunan Provincial (No. 2021JJ40956 and No. 2020JJ5851), the Hunan Science and Technology Department (No. 2020 SK4017), National Key Research and Development Program of China (No. 2018YFC1004800) and the Hunan Provincial Clinical Medical Technology Innovation Guiding Project (No. 2020SK53605). The authors have no other conflicts of interest to declare.

Ethical Statement: The authors are accountable for all aspects of the work in ensuring that questions related to the accuracy or integrity of any part of the work are appropriately investigated and resolved. Written informed consent for their medical records used for scientific 
research was signed voluntarily by each patient, and the ethics committee approved the study of the Third Xiangya Hospital of Central South University (IRB No. I-21046). The study was conducted in accordance with the Declaration of Helsinki (as revised in 2013).

Open Access Statement: This is an Open Access article distributed in accordance with the Creative Commons Attribution-NonCommercial-NoDerivs 4.0 International License (CC BY-NC-ND 4.0), which permits the noncommercial replication and distribution of the article with the strict proviso that no changes or edits are made and the original work is properly cited (including links to both the formal publication through the relevant DOI and the license). See: https://creativecommons.org/licenses/by-nc-nd/4.0/.

\section{References}

1. Zhao Y, Luo Q, Zhang X, Qin Y, Hao J, Kong D, Wang H, Li G, Gu X, Wang H. Clinical Efficacy and Safety of Stem Cell-Based Therapy in Treating Asherman Syndrome: A System Review and Meta-Analysis. Stem Cells Int 2020;2020:8820538.

2. Yan L, Wang A, Bai R, Shang W, Zhao Y, Wang W, Guo W. Application of SonoVue combined with three-dimensional color power angiography in the diagnosis and prognosis evaluation of intrauterine adhesion. Eur J Obstet Gynecol Reprod Biol 2016;198:68-72.

3. Mahdavinezhad F, Gharaei R, Farmani AR, Hashemi F, Kouhestani M, Amidi F. The Potential Relationship Between Different Human Female Reproductive Disorders and Sperm Quality in Female Genital Tract. Reprod Sci 2021. [Epub ahead of print].

4. Wu C, Dong Y, Li Y, Liu H. The efficacy of amniotic membrane-mediated sequential double-barrier therapy for the treatment of postoperative intrauterine adhesions. Medicine (Baltimore) 2021;100:e25416.

5. Zhao J, Chen Q, Cai D, Duan Z, Li X, Xue X. Dominant factors affecting reproductive outcomes of fertility-desiring young women with intrauterine adhesions. Arch Gynecol Obstet 2017;295:923-7.

6. Carugno J, Laganà AS, Vitale SG. Use of 3D ultrasound in the hysteroscopic management of Asherman syndrome. Ann Transl Med 2020;8:847.

7. Yang L, Wang L, Chen Y, Guo X, Miao C, Zhao Y, Li L, Zhang Q. Cold scissors versus electrosurgery for hysteroscopic adhesiolysis: A meta-analysis. Medicine (Baltimore) 2021;100:e25676.
8. Burjoo A, Zhao X, Zou L, Liu X, Lei L, Zhang B, Xu D. The role of preoperative 3D-ultrasound in intraoperative judgement for hysteroscopic adhesiolysis. Ann Transl Med 2020;8:55.

9. Cao M, Pan Y, Zhang Q, You D, Feng S, Liu Z. Predictive value of live birth rate based on different intrauterine adhesion evaluation systems following TCRA. Reprod Biol Endocrinol 2021;19:13.

10. Kim MJ, Lee Y, Lee C, Chun S, Kim A, Kim HY, Lee JY. Accuracy of three dimensional ultrasound and treatment outcomes of intrauterine adhesion in infertile women. Taiwan J Obstet Gynecol 2015;54:737-41.

11. Pleş L, Alexandrescu C, Ionescu CA, Arvătescu CA, Vladareanu S, Moga MA. Three-dimensional scan of the uterine cavity of infertile women before assisted reproductive technology use. Medicine (Baltimore) 2018;97:e12764.

12. Deans R, Vancaillie T, Ledger W, Liu J, Abbott JA. Live birth rate and obstetric complications following the hysteroscopic management of intrauterine adhesions including Asherman syndrome. Hum Reprod 2018;33:1847-53.

13. Chen L, Xiao S, He S, Tian Q, Xue M. Factors That Impact Fertility after Hysteroscopic Adhesiolysis for Intrauterine Adhesions and Amenorrhea: A Retrospective Cohort Study. J Minim Invasive Gynecol 2020;27:54-9.

14. Zhu R, Duan H, Wang S, Gan L, Xu Q, Li J. Decision Tree Analysis: A Retrospective Analysis of Postoperative Recurrence of Adhesions in Patients with Moderate-toSevere Intrauterine. Biomed Res Int 2019;2019:7391965.

15. Thomson AJ, Abbott JA, Deans R, Kingston A, Vancaillie TG. The management of intrauterine synechiae. Curr Opin Obstet Gynecol 2009;21:335-41.

16. Zhao X, Gao B, Yang X, Zhang A, Jamail G, Li Y, Xu D. The density of endometrial glandular openings: a novel variable to predict the live birth rate in patients with intrauterine adhesions following hysteroscopic adhesiolysis. Hum Reprod 2021;36:965-75.

17. Barel O, Krakov A, Pansky M, Vaknin Z, Halperin R, Smorgick N. Intrauterine adhesions after hysteroscopic treatment for retained products of conception: what are the risk factors? Fertil Steril 2015;103:775-9.

18. Nasr AL, Al-Inany HG, Thabet SM, Aboulghar M. A clinicohysteroscopic scoring system of intrauterine adhesions. Gynecol Obstet Invest 2000;50:178-81.

19. Valle RF, Sciarra JJ. Intrauterine adhesions: hysteroscopic diagnosis, classification, treatment, and reproductive outcome. Am J Obstet Gynecol 1988;158:1459-70. 
20. March CM, Israel R, March AD. Hysteroscopic management of intrauterine adhesions. Am J Obstet Gynecol 1978;130:653-7.

21. Ozdemir O, Sari ME, Kalkan D, Koc EM, Ozdemir S, Atalay CR. Comprasion of ovarian stromal blood flow measured by color Doppler ultrasonography in polycystic ovary syndrome patients and healthy women with ultrasonographic evidence of polycystic. Gynecol Endocrinol 2015;31:322-6.

22. Knopman J, Copperman AB. Value of 3D ultrasound in the management of suspected Asherman's syndrome. J Reprod Med 2007;52:1016-22.

23. Ni J, Han B, Liang J, Wang F. Three-dimensional 3D ultrasound combined with power Doppler for the differential diagnosis of endometrial lesions among infertile women. Int J Gynaecol Obstet 2019;145:212-8.

24. Doroftei B, Dabuleanu AM, Ilie OD, Maftei R, Anton E, Simionescu G, Matei T, Armeanu T. Mini-Review of the New Therapeutic Possibilities in Asherman SyndromeWhere Are We after One Hundred and Twenty-Six Years? Diagnostics (Basel) 2020;10:706.

25. Sanad AS, Aboulfotouh ME. Hysteroscopic adhesiolysis: efficacy and safety. Arch Gynecol Obstet 2016;294:411-6.

26. Yang JH, Chen CD, Chen SU, Yang YS, Chen MJ.

Cite this article as: Zhao X, Yang Y, Liao D, Traoré A, $\mathrm{He} \mathrm{S}, \mathrm{Xu}$ D. Correlative study of preoperative threedimensional transvaginal ultrasound findings and ongoing pregnancy/live birth in patients with intrauterine adhesions following hysteroscopic adhesiolysis: a retrospective study. Quant Imaging Med Surg 2022;12(4):2441-2453. doi: 10.21037/ qims-21-727
The influence of the location and extent of intrauterine adhesions on recurrence after hysteroscopic adhesiolysis. BJOG 2016;123:618-23.

27. Madani T, Ashrafi M, Abadi AB, Kiani K. Appropriate timing of uterine cavity length measurement positively affects assisted reproduction cycle outcome. Reprod Biomed Online 2009;19:734-6.

28. Lambers MJ, Dogan E, Lens JW, Schats R, Hompes PG. The position of transferred air bubbles after embryo transfer is related to pregnancy rate. Fertil Steril 2007;88:68-73.

29. van Gestel I, IJland MM, Hoogland HJ, Evers JL. Endometrial wave-like activity in the non-pregnant uterus. Hum Reprod Update 2003;9:131-8.

30. Jinno M, Ozaki T, Iwashita M, Nakamura Y, Kudo A, Hirano H. Measurement of endometrial tissue blood flow: a novel way to assess uterine receptivity for implantation. Fertil Steril 2001;76:1168-74.

31. Gu P, Yang X, Zhao X, Xu D. The value of transvaginal 4-dimensional hysterosalpingo-contrast sonography in predicting the necessity of assisted reproductive technology for women with tubal factor infertility. Quant Imaging Med Surg 2021;11:3698-714. 